\title{
Minimally Invasive Function-Preserving Gastrectomy with Sentinel Node Biopsy for Early Gastric Cancer
}

\author{
Yoshihiro Hiramatsu $^{\mathrm{a}, \mathrm{b}}$ Hiroya Takeuchi ${ }^{\mathrm{a}}$ Osamu Goto ${ }^{\mathrm{c}}$ Hirotoshi Kikuchi ${ }^{\mathrm{a}}$ \\ Yuko Kitagawa $^{\text {d }}$ \\ a Department of Surgery, Hamamatsu University School of Medicine, Hamamatsu-shi, Japan; ${ }^{b}$ Department \\ of Perioperative Functioning Care and Support, Hamamatsu University School of Medicine, Hamamatsu-shi, \\ Japan; 'Department of Gastroenterology, Nippon Medical School, Graduate School of Medicine, Tokyo, Japan; \\ ${ }^{\mathrm{d}}$ Department of Surgery, Keio University School of Medicine, Tokyo, Japan
}

\section{Keywords}

Sentinel node · Gastric cancer · Laparoscopic · Non-exposed endoscopic wall-inversion surgery

\begin{abstract}
Background: Recently, minimally invasive approaches such as endoscopic treatment or laparoscopic gastrectomy for early gastric cancer have made rapid progress. However, the extent of gastrectomy and lymphadenectomy in laparoscopic surgery is radical, similar to that of open gastrectomy for patients with early gastric cancer diagnosed as not indicated for endoscopic treatment. Since it is well known that lymph node metastasis is an important prognostic factor, the standard procedure of gastrectomy with radical lymph node dissection has been performed for the purpose of curative resection. The frequency of lymph node metastases is relatively low in patients with early gastric cancer; therefore, function-preserving gastrectomy, a solution between endoscopic treatment and standard gastrectomy in terms of invasiveness, could be considered to avoid excessive invasive surgery. Summary: A prospective multicenter trial and meta-analyses of sentinel node (SN) mapping and biopsy for early gastric cancer demonstrated favorable SN detection
\end{abstract}

rates and accuracy of nodal metastatic status. Personalized function-preserving gastrectomy with limited lymphadenectomy using SN theory will positively impact patients' quality of life (QOL). Specifically, a full-thickness partial gastrectomy by laparoscopic endoscopic cooperative surgery with $\mathrm{SN}$ basin dissection could become a reliable technique of minimally invasive gastrectomy for treating patients with clinically node-negative (cNO) early gastric cancer. Key Messages: For early gastric cancer, the development of laparoscopic personalized minimized gastrectomy with SN navigation may improve patients' postoperative QOL.

(c) 2018 S. Karger AG, Basel

\section{Introduction}

Recently, surgical treatment options for malignant tumors are increasing rapidly due to the improvement of diagnostic technology or the refinement of surgical procedures and devices. Endoscopic submucosal dissection (ESD) has also been performed as a less invasive treatment for $\mathrm{cT} 1$ gastric cancer. However, standard gastrectomy with radical lymphadenectomy is generally performed for patients with early gastric cancer diagnosed as

\section{KARGER}

(c) 2018 S. Karger AG, Basel

E-Mail karger@karger.com

www.karger.com/dig
Hiroya Takeuchi, MD

Department of Surgery, Hamamatsu University School of Medicine

1-20-1 Handayama, Higashi-ku

Hamamatsu-shi, Shizuoka 431-3192 (Japan)

E-Mail takeuchi@hama-med.ac.jp 
not having an indication for ESD [1]. Although laparoscopic gastrectomy is an intermediately invasive option between ESD and open gastrectomy, patients' quality of life (QOL) is mainly affected by post-gastrectomy syndromes after laparoscopic or open gastrectomy. The development of novel function-preserving, less invasive gastrectomy is essential to reduce perioperative complications and to improve patients' QOL in addition to radical curative resection [2].

\section{Sentinel Node Concept}

The sentinel node (SN) is considered as the first lymph node(s) receiving lymphatic drainage from the primary tumor site and is regarded as the first possible node(s) of metastasis from the primary lesion $[3,4]$. Theoretically, if SNs are pathologically negative for cancer metastasis, all regional lymph nodes can be predicted as negative for metastasis. Therefore, unnecessary radical lymphadenectomy can be avoided by intraoperative SN biopsy.

In 1992, SN navigation surgery was first applied to melanoma patients using patent blue as a tracer [4]. Minimally invasive surgery performed in accordance with the $\mathrm{SN}$ concept can prevent various complications related to unnecessary radical lymph node dissection in cases negative for SN metastasis. The SN concept was subsequently extended to patients with breast cancer or other solid tumors [5-6]. Several studies investigating the SN concept for early gastric cancer showed acceptable SN detection rates and accuracy to predict nodal metastatic status [3, 7]. A large-scale meta-analysis, consisting of 38 studies including 2,128 patients with early gastric cancer, showed that the SN detection rate and accuracy of nodal status diagnosis were 94 and 92\%, respectively [8].

Recently, a study group from the Japan Society of Sentinel Node Navigation Surgery (JSNNS) conducted a prospective multicenter trial (UMIN ID: 000000476) to demonstrate the feasibility of SN mapping and biopsy in early gastric cancer [9]. In this study, SN mapping was performed by using dual tracer technique for 397 patients with untreated $\mathrm{CT} 1$ or $\mathrm{CT} 2$ gastric cancer with primary lesion $<4 \mathrm{~cm}$ in gross diameter. Following biopsy of the identified SNs, standardized D2 or modified D2 gastrectomy was performed according to current Japanese Gastric Cancer Association guidelines to clarify the accuracy of $\mathrm{SN}$ mapping. The study resulted in a $\mathrm{SN}$ detection rate of $97.5 \%$ and an accuracy for nodal metastatic status of $99.0 \%$. False-negative SN biopsy was observed only in $1.0 \%$ ( 4 of 397), and 3 of 4 patients had primary tumors

Function-Preserving Gastrectomy for Early Gastric Cancer with pT2 or $>4 \mathrm{~cm}$ in size. The study showed that SN mapping for gastric cancer is feasible, reliable, and provides the possibility of using precision medicine based on SN mapping for early gastric cancer. Based on these, a prospective multicenter trial (UMIN ID: 000014401) including our institute about the development of novel strategies by the combination of laparoscopic functionpreserving gastrectomy and SN mapping was performed to evaluate the recurrence-free survival, overall survival, and patients' QOL after surgery. This study included clinical T1N0M0 early gastric cancers without indication for ESD with a single primary lesion of less than $40 \mathrm{~mm}$ in size to avoid false-negative SN (Fig. 1). In addition, in case of patients with a large primary lesion, it may be difficult to perform stomach-preserving gastrectomy without a postoperative deformity of the remaining stomach. In this study, $\mathrm{SN}$ basin dissection, which is a selective lymphadenectomy for en bloc dissection of lymphatic tissue including lymph nodes and vessels, was performed even in patients without SN metastasis to secure the curability of the surgery.

\section{Laparoscopic SN Mapping Procedures}

The dual-tracer method using both blue or green dye and radioactive colloids is considered the standard procedure in early gastric cancer patients $[2,9]$. The blue or green dye is useful to visualize the lymphatic flow even during laparoscopic surgery. In addition, the accumulation of radioactive colloids in SNs enables the identification of SNs using hand-held gamma probes even in resected specimens. Tecnetium-99m colloids (tin, sulfur, and antibody sulfur) are preferentially used as radioactive tracers. As dye tracers, isosulfan blue, indocyanine green (ICG), and patent blue are the most frequent options. Moreover, with the recent invention of devices such as the Photodynamic Eye (Hamamatsu Photonics, Hamamatsu, Japan), HyperEye Medical System (Mizuho Medical, Tokyo, Japan), and VISERA ELITE II (Olympus, Tokyo, Japan), ICG fluorescence imaging for SN mapping is currently being used in patients with various types of cancer [10-14].

In our institution, the day before surgery, $2 \mathrm{~mL}(150$ $\mathrm{MBq}$ ) of technetium-99m tin colloid solution is endoscopically injected into 4 quadrants of the submucosal layer of the primary lesion by using an endoscopic puncture needle. Intraoperatively, the $0.5 \%$ ICG solution is also injected in the submucosal layer surrounding the primary lesion in the same manner to function as the radio- 
Fig. 1. Study scheme of the clinical trial for laparoscopic function-preserving gastrectomy with SN mapping. SN, sentinel node; $\mathrm{ESD}$, endoscopic submucosal dissection.

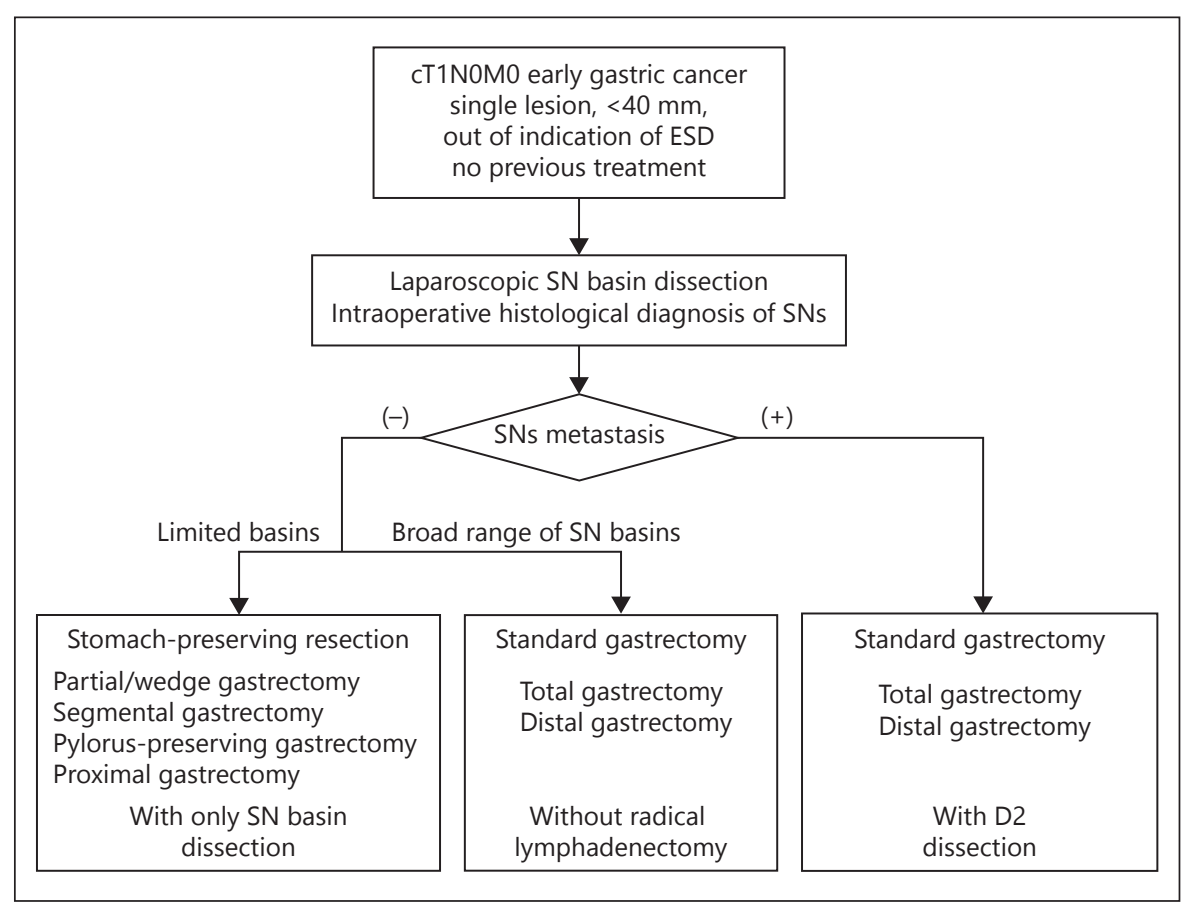

active tracer (Fig. 2). Within 15 min after the injection of ICG solution, the lymphatic vessels and lymph nodes are dyed green and visualized. Simultaneously, ICG fluorescence in the lymphatic vessels and lymph nodes is also detected with VISERA ELITE II during laparoscopic operation (Fig. 3). ICG fluorescence imaging enables the easy visualization of $\mathrm{SNs}$ and will replace the $\mathrm{SN}$ mapping method in many malignancies including gastric cancer [15-17]. Subsequently, a laparoscopic gamma probe introduced from the trocar port is used to locate the radioactive SN. Lymph nodes with radioactivity increased more than 10 times when compared with environmental radioactivity, and are defined as hot nodes. The hot nodes identified by a gamma probe and positive nodes by fluorescence observation are identified as SNs.

\section{Laparoscopic SN Basin Dissection and Precision Medicine}

After SN mapping, SN basin dissection is performed. The gastric lymphatic basins are divided into 5 areas along the main arteries as follows: left gastric artery, left gastroepiploic artery, right gastroepiploic artery, right gastric artery, and posterior gastric artery [18]. Harvested SNs are confirmed by fluorescence imaging and gamma probe in resected basins on the back table and subjected

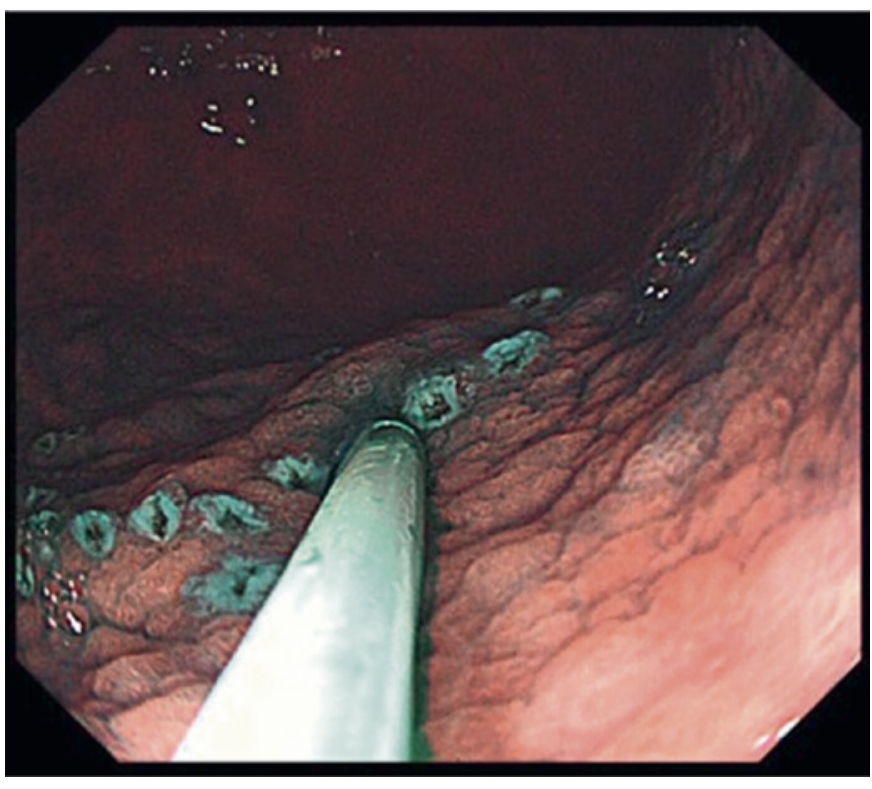

Fig. 2. Intraoperative endoscopic submucosal injection of ICG surrounding the primary lesion. ICG, indocyanine green.

to intraoperative histological examination. Pathological status of SNs and distribution of SN basins provide the necessary information for using precision medicine. If SNs are positive for lymphatic metastasis, standard gastrectomy with radical lymph nodes dissection is per-
16
Hiramatsu/Takeuchi/Goto/Kikuchi/ Kitagawa 

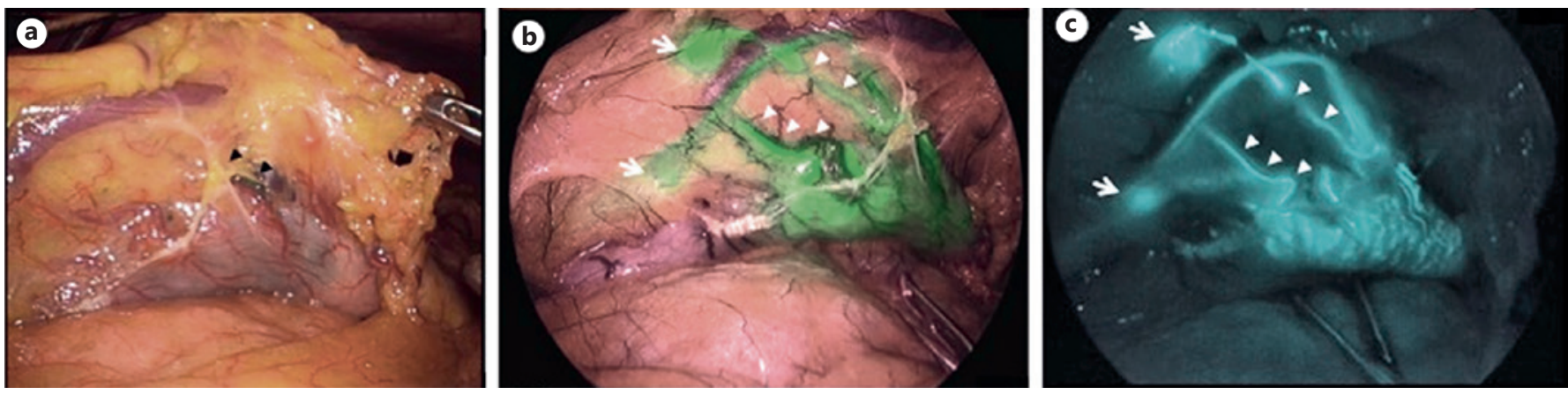

Fig. 3. Intraoperative pictures of SN mapping (arrows: SNs, arrowheads: lymphatic vessels). a Sentinel lymphatic staining with ICG under ordinary laparoscopy. b ICG fluorescence image of the same area. c Observation under illumination with infrared ray electronic endoscopy. SN, sentinel node; ICG, indocyanine green.

Fig. 4. Laparoscopic function-preserving gastrectomy with SNBD. a Partial gastrectomy with SNBD. b Segmental gastrectomy with SNBD. c Proximal gastrectomy with SNBD and D1+ lymphadenectomy. SNDB, sentinel node basin dissection.

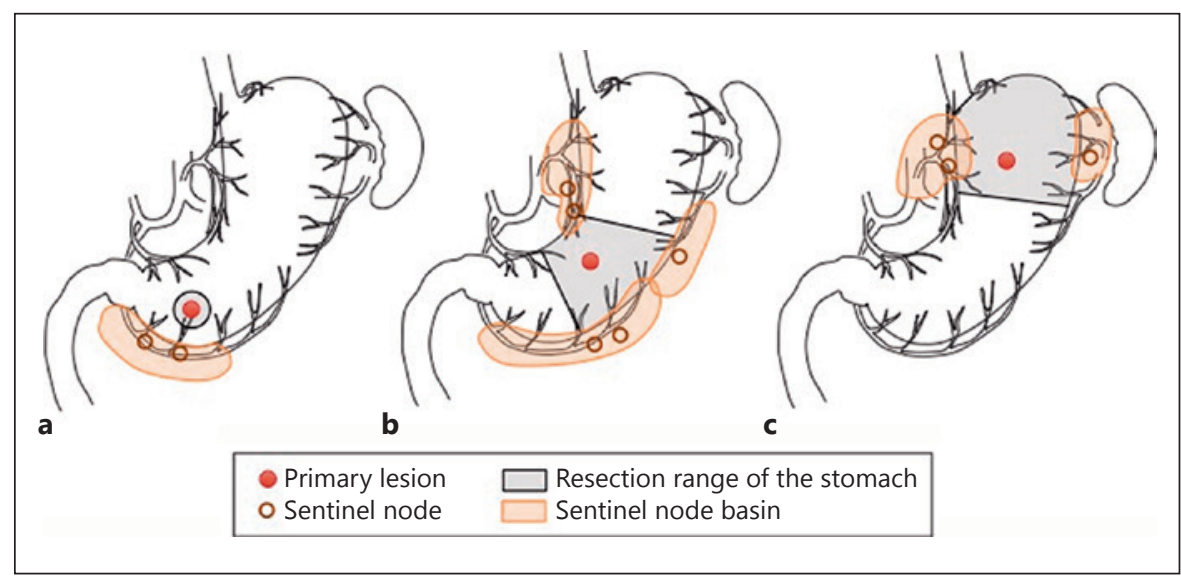

formed. On the contrary, if SNs are negative for metastasis, function-preserving gastrectomy with $\mathrm{SN}$ basin dissection, including partial resection, segmental gastrectomy, pylorus-preserving gastrectomy, and proximal gastrectomy would be possible for each patient (Fig. 4) [19, 20]. Laparoscopic minimally invasive function-preserving gastrectomy using $\mathrm{SN}$ navigation surgery is expected to contribute to the improvement of longterm patients' QOL.

In cases of laparoscopic partial gastrectomy, laparoscopic and endoscopic cooperative surgeries are useful in the determination of the stomach resection line since the demarcation line of the primary tumor cannot be recognized accurately from the outside of the stomach $[21,22]$. However, in cancer cases, the full-thickness resection techniques without transluminal access seem to be important to avoid the risk of tumor seeding [23]. Non-exposed endoscopic wall-inversion surgery (NEWS) was developed as a new useful technique of endoscopic full-

Function-Preserving Gastrectomy for Early Gastric Cancer thickness resection of the stomach without intentional perforation for early gastric cancer (Fig. 5) [24]. In an ongoing multicenter prospective clinical trial (UMIN ID: 000014401), treatment data of non-exposed endoscopic wall-inversion surgery and $\mathrm{SN}$ basin dissection combination have been collected [25]. This strategy is considered to be a promising, ideal, minimally invasive, functionpreserving surgical treatment for patients with cT1N0 early gastric cancer.

\section{Present Status and Future Prospects of SN Navigation Surgery for Early Gastric Cancer}

The JSNNS study showed that SN biopsy using the endoscopic dual tracer method was safe and effective for the treatment of patients with superficial, relatively small gastric cancers [9]. Ichikura et al. [26] reported that limited gastrectomy with dissection of SN stations for T1N0 gas- 

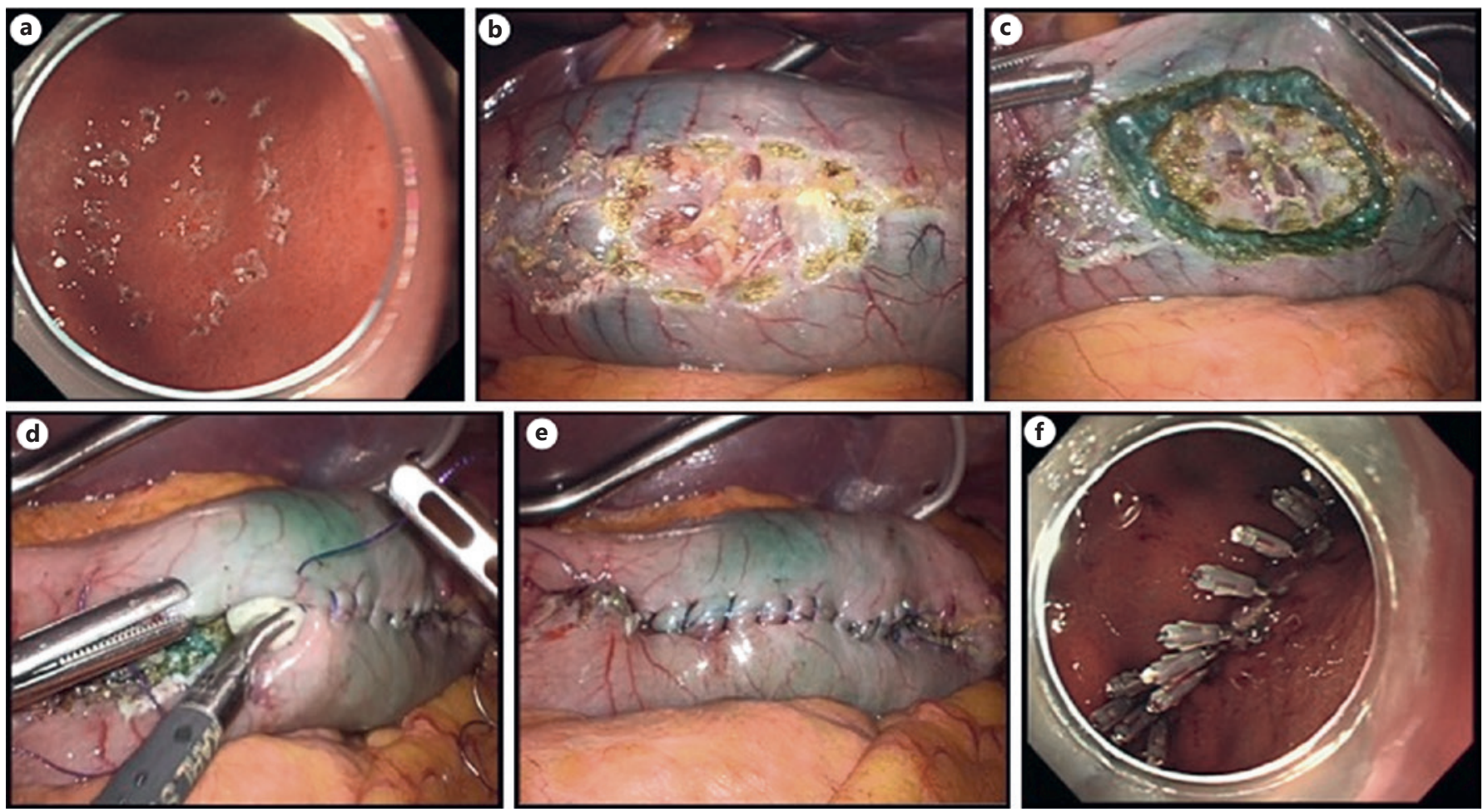

Fig. 5. Intraoperative pictures of non-exposed endoscopic wallinversion surgery (NEWS). a Mucosal markings were endoscopically made around the primary lesion. b Serosal markings were made by electric scalpel. c Laparoscopic circumferential seromus-

tric cancer was considered safe and acceptable. In contrast, the Japan Clinical Oncology Group (JCOG) also conducted a multicenter prospective trial (JCOG0302) of SN biopsy for cT1N0 gastric cancer [27]. This study was designed to evaluate the feasibility and accuracy of diagnosis using SN biopsy with intraoperative subserosal injection of ICG solution. Patient recruitment was suspended when 440 patients were enrolled because the proportion of false negatives was high. Several causes were involved, including technical problems of the dye-only guided technique with subserosal injection. It might be critical that only 5 cases were required as the minimum experience for the initial learning phase to participate in this study [27].

Similar to our multicenter prospective ongoing clinical trial to assess the long-term survival and postoperative patients' QOL after laparoscopic function-preserving gastrectomy with SN mapping and SN basin dissection, a Korean group has also conducted a prospective multicenter randomized controlled phase III clinical trial (SENORITA trial) to elucidate the oncological safety of laparoscopic stomach-preserving surgery with SN basin cular incision surrounding the primary tumor. $\mathbf{d}$ and e Laparoscopic seromuscular suturing and inversion of the primary tumor site to the inside of the stomach. $\mathbf{f}$ Endoscopic mucosal closure using endoscopic clips. dissection compared to a standard laparoscopic gastrectomy [28]. These proposed trials are expected to verify whether laparoscopic limited gastrectomy with SN basin dissection achieves similar oncological outcomes and improves patients' QOL compared to a standard gastrectomy with radical lymphadenectomy for early gastric cancer patients. A combination of ESD and laparoscopic SN biopsy is another potential option as a novel, whole stomach-preserving minimally invasive treatment when all SNs are pathologically negative for metastasis. In the SENORITA trial, if all the harvested SNs are tumor-free, stomach-preserving primary tumor resection is carried out, including ESD, partial gastrectomy, and segmental gastrectomy. On the contrary, it remains controversial whether SN mapping would be feasible after ESD because the lymphatic flow from the primary tumor site where ESD is performed remains unknown. However, a previous study suggested that at least the SN basin was not markedly changed in patients who underwent ESD prior to surgery $[29,30]$. Regarding the combination treatment of ESD and SN biopsy, further studies are required to confirm safety and efficacy.
Hiramatsu/Takeuchi/Goto/Kikuchi/ Kitagawa 
Results of ongoing Japanese and Korean trials would be expected to establish the ideal, personalized, minimally invasive function-preserving treatment to improve the patients' postoperative QOL with sufficient long-time survival after surgery.

\section{Conclusion}

For patients with early gastric cancer, good prognosis is achieved by conventional radical gastrectomy. However, conventional gastrectomy often decreases patients' QOL postoperatively due to post-gastrectomy syn- drome. In the future, we believe that $\mathrm{SN}$ theory could become the ideal treatment option for early gastric cancer patients.

\section{Disclosure Statement}

The authors have no conflicts of interest to disclose.

\section{Funding Sources}

The present study was supported by Japan Agency for Medical Research and Development (AMED) under Grant Number JP18ck0106300.

\section{References}

1 Kato $\mathrm{H}$, Ono $\mathrm{H}$, Hamamoto $\mathrm{Y}$, Ishikawa $\mathrm{H}$ : Interaction between medical treatment and minimally invasive surgical treatment for the malignancies of the digestive tract. Digestion 2018;97:13-19.

2 Takeuchi H, Kitagawa Y: New sentinel node mapping technologies for early gastric cancer. Ann Surg Oncol 2013;20:522-532.

3 Kitagawa Y, Fujii H, Mukai M, Kubota T, Ando N, Watanabe M, Ohgami M, Otani Y, Ozawa S, Hasegawa H, Furukawa T, Kumai K, Ikeda T, Nakahara T, Kubo A, Kitajima M: The role of the sentinel lymph node in gastrointestinal cancer. Surg Clin North Am 2000; 80:1799-1809.

4 Morton DL, Wen DR, Wong JH, Economou JS, Cagle LA, Storm FK, Foshag LJ, Cochran AJ: Technical details of intraoperative lymphatic mapping for early stage melanoma. Arch Surg 1992;127:392-399.

5 Giuliano AE, Kirgan DM, Guenther JM, Morton DL: Lymphatic mapping and sentinel lymphadenectomy for breast cancer. Ann Surg 1994;220:391-401.

6 Bilchik AJ, Saha S, Wiese D, Stonecypher JA, Wood TF, Sostrin S, Turner RR, Wang HJ, Morton DL, Hoon DS: Molecular staging of early colon cancer on the basis of sentinel node analysis: a multicenter phase II trial. J Clin Oncol 2001;19:1128-1136.

7 Miwa K, Kinami S, Taniguchi K, Fushida S, Fujimura T, Nonomura A: Mapping sentinel nodes in patients with early-stage gastric carcinoma. Br J Surg. 2003;90:178-182.

8 Wang Z, Dong ZY, Chen JQ, Liu JL: Diagnostic value of sentinel lymph node biopsy in gastric cancer: a meta-analysis. Ann Surg Oncol 2012;19:1541-1550.

9 Kitagawa Y, Takeuchi H, Takagi Y, Natsugoe S, Terashima M, Murakami N, Fujimura T, Tsujimoto H, Hayashi H, Yoshimizu N, Takagane A, Mohri Y, Nabeshima K, Uenosono Y, Kinami S, Sakamoto J, Morita S, Aikou T, Miwa K, Kitajima M: Sentinel node mapping for gastric cancer: a prospective multicenter trial in Japan. J Clin Oncol 2013;31:37043710 .

10 Miyashiro I, Miyoshi N, Hiratsuka M, Kishi K, Yamada T, Ohue M, Ohigashi H, Yano M, Ishikawa O, Imaoka S: Detection of sentinel node in gastric cancer surgery by indocyanine green fluorescence imaging: comparison with infrared imaging. Ann Surg Oncol 2008;15: 1640-1643.

11 Yoshida M, Kubota K, Kuroda J, Ohta K, Nakamura T, Saito J, Kobayashi M, Sato T, Beck Y, Kitagawa Y, Kitajima M: Indocyanine green injection for detecting sentinel nodes using color fluorescence camera in the laparoscopy-assisted gastrectomy. J Gastroenterol Hepatol 2012;27(suppl 3):S29-S33.

12 Kubota K, Yoshida M, Kuroda J, Okada A, Ohta K, Kitajima M: Application of the HyperEye medical system for esophageal cancer surgery: a preliminary report. Surg Today 2013;43:215-220.

13 Aoyama K, Kamio T, Ohchi T, Nishizawa M, Kameoka S: Sentinel lymph node biopsy for breast cancer patients using fluorescence navigation with indocyanine green. World J Surg Oncol 2011;9:157.

14 Schaafsma BE, van der Vorst JR, Gaarenstroom KN, Peters AA, Verbeek FP, de Kroon CD, Trimbos JB, van Poelgeest MI, Frangioni JV, van de Velde CJ, Vahrmeijer AL: Randomized comparison of near-infrared fluorescence lymphatic tracers for sentinel lymph node mapping of cervical cancer. Gynecol Oncol 2012;127:126-130.

15 Tajima Y, Murakami M, Yamazaki K, Masuda Y, Kato M, Sato A, Goto S, Otsuka K, Kato T, Kusano M: Sentinel node mapping guided by indocyanine green fluorescence imaging during laparoscopic surgery in gastric cancer. Ann Surg Oncol 2010;17:1787-1793.

16 Ishikawa K, Yasuda K, Shiromizu A, Etoh T, Shiraishi N, Kitano S: Laparoscopic sentinel node navigation achieved by infrared ray elec- tronic endoscopy system in patients with gastric cancer. Surg Endosc 2007;21:1131-1134.

17 Kinami S, Oonishi T, Fujita J, Tomita Y, Funaki $H$, Fujita $H$, Nakano Y, Ueda N, Kosaka $\mathrm{T}$ : Optimal settings and accuracy of indocyanine green fluorescence imaging for sentinel node biopsy in early gastric cancer. Oncol Lett 2016;11:4055-4062.

18 Kinami S, Fujimura T, Ojima E, Fushida S, Ojima T, Funaki H, Fujita H, Takamura H, Ninomiya I, Nishimura G, Kayahara M, Ohta T, Yoh Z: PTD classification: proposal for a new classification of gastric cancer location based on physiological lymphatic flow. Int J Clin Oncol 2008;13:320-329.

19 Takeuchi H, Saikawa Y, Kitagawa Y: Laparoscopic sentinel node navigation surgery for early gastric cancer. Asian J Endosc Surg 2009;2:13-17.

20 Takeuchi H, Oyama T, Kamiya S, Nakamura R, Takahashi T, Wada N, Saikawa Y, Kitagawa Y: Laparoscopy-assisted proximal gastrectomy with sentinel node mapping for early gastric cancer. World J Surg 2011;35:2463-2471.

21 Hiki N, Yamamoto Y, Fukunaga T, Yamaguchi T, Nunobe S, Tokunaga M, Miki A, Ohyama S, Seto Y: Laparoscopic and endoscopic cooperative surgery for gastrointestinal stromal tumor dissection. Surg Endosc 2008;22: 1729-1735.

22 Nunobe S, Hiki N, Gotoda T, Murao T, Haruma K, Matsumoto H, Hirai T, Tanimura S, Sano T, Yamaguchi T: Successful application of laparoscopic and endoscopic cooperative surgery (LECS) for a lateral-spreading mucosal gastric cancer. Gastric Cancer 2012;15: 338-342.

23 Mori H, Rahman A, Kobara H, Fujihara S, Nishiyama N, Ayaki M, Matsunaga T, Murakami M, Masaki T: Current status of exposed endoscopic full-thickness resection and further development of non-exposed endoscopic full-thickness resection. Digestion 2017;95:6-15. 
24 Goto O, Takeuchi H, Kawakubo H, Sasaki M, Matsuda T, Matsuda S, Kigasawa Y, Kadota Y, Fujimoto A, Ochiai Y, Horii J, Uraoka T, Kitagawa Y, Yahagi N: First case of non-exposed endoscopic wall-inversion surgery with sentinel node basin dissection for early gastric cancer. Gastric Cancer 2015;18:434-439.

25 Takeuchi H, Kitagawa Y: Sentinel lymph node biopsy in gastric cancer. Cancer J 2015;21:2124.

26 Ichikura T, Sugasawa H, Sakamoto N, Yaguchi $\mathrm{Y}$, Tsujimoto $\mathrm{H}$, Ono S: Limited gastrectomy with dissection of sentinel node stations for early gastric cancer with negative sentinel node biopsy. Ann Surg 2009;249: 942-947.
27 Miyashiro I, Hiratsuka M, Sasako M, Sano T, Mizusawa J, Nakamura K, Nashimoto A, Tsuburaya A, Fukushima N; Gastric Cancer Surgical Study Group (GCSSG) in the Japan Clinical Oncology Group (JCOG): High false-negative proportion of intraoperative histological examination as a serious problem for clinical application of sentinel node biopsy for early gastric cancer: final results of the Japan Clinical Oncology Group multicenter trial JCOG0302. Gastric Cancer 2014; 17:316-323.

28 Park JY, Kim YW, Ryu KW, Nam BH, Lee YJ, Jeong SH, Park JH, Hur H, Han SU, Min JS, An JY, Hyung WJ, Cho GS, Jeong GA, Jeong O, Park YK, Jung MR, Yoon HM, Eom BW:
Assessment of laparoscopic stomach preserving surgery with sentinel basin dissection versus standard gastrectomy with lymphadenectomy in early gastric cancer - a multicenter randomized phase III clinical trial (SENORITA trial) protocol. BMC Cancer 2016; 16:340.

29 Takeuchi H, Kitagawa Y: Sentinel node navigation surgery in patients with early gastric cancer. Dig Surg 2013;30:104-111.

30 Mayanagi S, Takeuchi H, Kamiya S, Niihara M, Nakamura R, Takahashi T, Wada N, Kawakubo H, Saikawa Y, Omori T, Nakahara T, Mukai M, Kitagawa Y: Suitability of sentinel node mapping as an index of metastasis in early gastric cancer following endoscopic resection. Ann Surg Oncol 2014;21:2987-2993. 